\title{
Periodic-Neumann boundary value problem for nonlinear parabolic equations and application to an elliptic equation
}

\author{
by Juan J. Nieto (Santiago de Compostela)
}

\begin{abstract}
In this paper we study the periodic-Neumann boundary value problem for a class of nonlinear parabolic equations. We prove a new uniqueness result and study the structure of the set of solutions when there exist more than one solution. The ideas are applied to a Neumann problem for an elliptic equation.
\end{abstract}

1. Introduction. In this paper we study the existence and uniqueness of periodic solutions for a parabolic equation. When there exist more than one solution we study the structure of the set of solutions.

The existence and multiplicity of periodic solutions of nonlinear parabolic equations is an important question and has been investigated by several authors (see, for instance, $[2,3,8,9,13,16]$ and the references therein). The present work is motivated by [13] where we study the structure of the set of solutions. Here, we continue that work and improve some results about the solution set when the nonlinearity is given by a decreasing function $g$. Moreover, we show that the conjecture mentioned in [13] is true provided that $g$ is Lipschitz continuous and the Lipschitz constant is less that one.

To this purpose we give a new uniqueness result (Theorem 1) and then prove our main result (Theorem 2): The solution set is nonempty, compact, connected and acyclic. As a consequence, we find that the set of solutions is a continuum (Corollary 3), that is, satisfies the classical Hukuhara-Kneser property for ordinary differential equations. We recall that this property is satisfied, for instance, by an initial value problem for a system of partial differential equations of parabolic type [5].

We mention that our method can be adapted, in a direct way, to the study of other boundary value problems. To illustrate this, we consider a Neumann problem and improve the result of [11].

1985 Mathematics Subject Classification: Primary 35K20. 
Finally, we present some problems for further research on the topic.

2. Preliminaries. We consider the following periodic boundary value problem (PBVP):

$$
\begin{aligned}
& u_{t}-u_{x x}+g(u)=h(t, x), \quad(t, x) \in \Omega=[0,2 \pi] \times[0, \pi], \\
& u(0, x)=u(2 \pi, x), \quad x \in J=[0, \pi], \\
& u_{x}(t, 0)=u_{x}(t, \pi)=0, \quad t \in I=[0,2 \pi],
\end{aligned}
$$

where $g \in C(\mathbb{R}, \mathbb{R})$ and $h \in C[\Omega, \mathbb{R}]$. Set $v=2 \pi^{2}$, Range $g=\{g(x): x \in \mathbb{R}\}$ and

$$
\omega=\omega(h)=\frac{1}{v} \int_{0}^{2 \pi} \int_{0}^{\pi} h(t, x) d x d t .
$$

We shall denote by $S=S(h)$ the set of solutions to (1). We list the following assumptions for convenience.

(A1) $g$ is a bounded and decreasing function.

(A2) $\omega \in \operatorname{Int}($ Range $g)$.

(A3) There exists $\gamma \in[0,1)$ and $C \in \mathbb{R}$ such that $|g(u)| \leq \gamma|u|+C$ for every $u \in \mathbb{R}$.

(A4) There exists $k \in[0,1)$ such that $|g(u)-g(v)| \leq k|u-v|$ for every $u, v \in \mathbb{R}$.

Note that (A3) is a more general assumption than (A4). Obviously, any bounded function satisfies (A3) with $\gamma=0$.

Let $E=\left\{u \in C^{1,2}[\Omega, \mathbb{R}]: u(0, x)=u(2 \pi, x), x \in J\right.$, and $u_{x}(t, 0)=$ $\left.u_{x}(t, \pi)=0, t \in I\right\}$ and $F=C[\Omega, \mathbb{R}]$. In $F$ we consider the norm $\|u\|=$ $\sup \{|u(t, x)|:(t, x) \in \Omega\}$, and in $E,\|u\|_{E}=\|u\|+\left\|u_{t}\right\|+\left\|u_{x x}\right\|$. Define the operator $L: E \rightarrow F$ by $L u=u_{t}-u_{x x}$, and let $P: F \rightarrow F$ be the projection defined by

$$
P u=\frac{1}{v} \int_{0}^{2 \pi} \int_{0}^{\pi} u(t, x) d x d t .
$$

Hence, $\operatorname{Ker} L=\langle 1\rangle$ and Range $L=\{v \in F: P v=0\}=(I-P) F$. As usual, we can write $E=E_{0} \oplus E_{1}, F=F_{0} \oplus F_{1}$ where $E_{0}=P E, F_{0}=P F$, and for $u \in E, u=u_{0}+u_{1}, u_{0} \in E_{0}, u_{1} \in E_{1}$. The partial inverse of $L$ is $H: F_{1} \rightarrow E_{1}$.

In $L^{2}(\Omega)$ we define the usual inner product and norm given by

$$
(u, v)=\int_{\Omega} u(t, x) v(t, x) d t d x \quad\|u\|_{2}=\sqrt{(u, u)} .
$$

We note that if (A1) and (A2) are satisfied, then $S(h)$ is bounded [13]. 
3. A uniqueness result. If $g$ is strictly decreasing uniqueness does not occur in general (see the example on page 97 in [13]). We now give a uniqueness result that improves $[13$, Th. 6]. In order to use the alternative method [6,7], define the nonlinear operator $N: E \rightarrow F$ by $N u=h-g(u)$. Thus, (1) is equivalent to the abstract equation $L u=N u$. Note that the solutions of this equation are precisely the fixed points of the compact operator $T: E \rightarrow E, T u=P u+H(I-P) N u+P N u$.

THEOREM 1. If (A1), (A2) and (A4) hold, and $g$ is strictly decreasing, then any solution of (1) is unique.

Proof. The values of $\lambda$ for which the problem

$$
-L u+\lambda u=-u_{t}+u_{x x}+\lambda u=0, \quad u \in E,
$$

has nontrivial solutions are $\lambda_{n}=(n-1)^{2}, n=1,2, \ldots$, and $\operatorname{Ker}\left(-L+\lambda_{n} I\right)=$ $\langle\cos (n-1) x\rangle($ see $[8])$.

Take $c=\left(\lambda_{1}+\lambda_{2}\right) / 2=1 / 2$. Thus the operator $-L+c I$ is invertible and $\left\|(-L+c I)^{-1}\right\| \leq c^{-1}[6$, p. 116]. On the other hand, for every $x, y \in \mathbb{R}$ we have $|g(x)-g(y)+c(x-y)| \leq c|x-y|$, with strict inequality when $x \neq y$ since $g$ is strictly decreasing and (A4) holds. Therefore, for every $u, v \in E$, $\|N v-N u+c(u-v)\| \leq c\|u-v\|$. Moreover, if $u \neq v$ (in a set of positive measure) then $\|N v-N u+c(u-v)\|<c\|u-v\|$.

Now, if $u, v$ are two solutions of $(1)$, then $(-L+c I)^{-1}[N v-N u+c(u-$ $v)]=u-v$, which implies that $\|u-v\| \leq c^{-1}\|N v-N u+c(u-v)\|$. In consequence, $u=v$ and (1) has at most one solution.

We note that the estimate $k<1$ is optimal since $\lambda_{2}=1$. However, the result of Theorem 1 is no longer true if we assume (A3) instead of (A4) (even with $\gamma=0$ ).

ExAMPLE. Take $n \in \mathbb{N}^{*}$ and let $g$ be a strictly decreasing and bounded function with $g(u)=-n^{2} u$ for $|u|<1$, and $h=0$. Thus, for $a$ small, $u(t, x)=a \cos n x$ is a solution of (1).

\section{Main result}

TheOrem 2. Assume that (A1), (A2) and (A4) are satisfied. Then the set of solutions of (1) is nonempty, compact, connected and acyclic.

Proof. Consider the sets $S_{0}=\{u \in \operatorname{Ker} L: N u \in$ Range $L\}$, and $S_{+}=\{u \in E: L u=\lambda N u$, for some $\lambda \in(0,1)\}$. As in [13] one can show that $S_{0}$ and $S_{+}$are bounded sets in $F$. Now, define $G: \mathbb{R} \rightarrow E$ and $G_{1}: F \rightarrow \mathbb{R}$ by $G(a)=a, G_{1}(u)=P u$ respectively. Thus $\xi(a)=G_{1} N G(a)=P[h-g(a)]$. Using (A2) we see that $\xi(a) \xi(-a)<0$ for $a>0$ large, which implies [4] that $S(h)$ is nonempty and compact in $E$. 
In order to show that the solution set is connected, define, for $n \in \mathbb{N}^{*}$, the function $g_{n}(u)=g(u)-(a n)^{-1} \arctan u$ with $a>(1-k)^{-1}$, and the operator $N_{n}: E \rightarrow F$ by $N_{n}(u)=h-g_{n}(u)$. Thus, $\left\|N_{n}(u)-N u\right\| \leq$ $(\text { an })^{-1}\|\arctan u\|$, which shows that $N_{n}$ converges to $N$ uniformly on $E$.

For $v \in S(h)$, consider the set $S_{n}(v)=\left\{u \in E: L u-N_{n} u=L v-N_{n} v\right\}$, and let $u \in S_{n}(v)$. Hence,

$$
u_{t}-u_{x x}+g_{n}(u)=v_{t}-v_{x x}+g_{n}(v), \quad u \in E .
$$

Note that $g_{n}$ is strictly increasing and satisfies (A4) since $\left|g_{n}(u)-g(u)\right| \leq$ $\left[k+(a n)^{-1}\right]|u-v|$, and $k+a^{-1}<1$. On the other hand,

$$
\begin{aligned}
\omega \in \operatorname{Int}\left(\text { Range } g_{n}\right) & =\left(g(\infty)-\pi(a n)^{-1}, g(-\infty)+\pi(a n)^{-1}\right) \\
& \subset(g(\infty), g(-\infty)) .
\end{aligned}
$$

In view of Theorem 1 we see that (2) has a unique solution and $S_{n}(v)$ is a singleton. In consequence, $S(h)$ is connected. Now, noting that for $v \in E$, the equation $L u-N_{n} u=v$ has at most one solution, we can conclude that $S(h)$ is acyclic. This completes the proof of the theorem.

We note that this result improves [13, Th. 7]. As a consequence we have proved the conjecture of [13] when (A4) holds. In addition, we obtain the classical Hukuhara-Kneser property for $S(h)$.

COROLlary 3 . Under the hypothesis of Theorem 2, the set of solutions of (1) is a continuum.

5. An elliptic problem. Using the same method, we can study some other boundary value problems. To illustrate this, consider the following Neumann problem [11]:

$$
-\Delta u+g(u)=h \quad \text { in } \Omega, \quad \partial u / \partial \nu=0 \quad \text { on } \partial \Omega,
$$

where $g$ is continuous and bounded, and $\Omega$ a bounded region in $\mathbb{R}^{n}$ with smooth boundary. The values of $\lambda$ for which $-\Delta u+\lambda u=0$ in $\Omega, \partial u / \partial \nu=0$ on $\partial \Omega$ has nontrivial solutions form an increasing sequence $\lambda_{n}, n=1,2, \ldots$, with $\lambda_{1}=0<\lambda_{2}$. The following result is analogous to Theorem 1 and improves the result of [11] since our result gives an optimal estimate for the Lipschitz constant (see the example in [11]).

Theorem 4. Let $\omega=\int_{\Omega} h(x) d x$. Suppose that $g$ is strictly decreasing and Lipschitz continuous with Lipschitz constant less than $\lambda_{2}$, and $\omega \in$ Int(Range $g$ ). Then (3) has a unique solution.

TheOREM 5. Assume that $g$ is decreasing, Lipschitz continuous with Lipschitz constant less than $\lambda_{2}$, and $\omega \in \operatorname{Int}(\operatorname{Range} g)$. Then the set of solutions of (3) is nonempty, compact, connected and acyclic. 
This last result improves [14, Th. 5] and proves the conjecture of [14] when the Lipschitz constant is less than $\lambda_{2}$.

6. Final remarks. If $g$ is increasing, (1) can be written as

$$
L u+N u=h
$$

with $L$ and $N$ monotone operators. Existence and uniqueness results are known for this equation. When uniqueness does not occur one can show that the solution set is a convex set by using the direct method of the calculus of variations combined with the principles of convex analysis [12], or an $R_{\delta}$ as in [15] by using the alternative method and Aronszajn's theorem. However, when $g$ is decreasing the techniques developed in those papers are not applicable to the PBVP (1). Note that (1) and (3), with $g$ decreasing, can be written as

$$
L u-M u=h
$$

with $L$ and $M$ monotone operators. We hope to give some general results about the structure of the solution set for equation $(* *)$. Finally, we think (inspired by the work of Ahmad [1]) that the following result holds true.

Conjecture. Let $g$ be continuous such that $-\infty \leq \overline{g(-\infty)}<\omega<$ $g(\infty) \leq \infty$, and (A3) holds. Then (1) is solvable.

\section{References}

[1] S. A hmad, A resonance problem in which the nonlinearity may grow linearly, Proc. Amer. Math. Soc. 92 (1984), 381-384.

[2] H. A mann, Periodic solutions of semilinear parabolic equations, in: Nonlinear Analysis, Academic Press, New York 1978, 1-29.

[3] D. W. Bange, Periodic solutions of a quasilinear parabolic differential equation, J. Differential Equations 17 (1975), 61-72.

[4] J. W. Bebernes and M. Martelli, On the structure of the solution set for periodic boundary value problems, Nonlinear Anal. 4 (1980), 821-830.

[5] J. W. Bebernes and K. Schmitt, Invariant sets and Hukuhara-Kneser property for systems of parabolic partial differential equations, Rocky Mountain J. Math. 7 (1977), 557-567.

[6] L. Cesari, Functional analysis, nonlinear differential equations and the alternative method, in: Nonlinear Functional Analysis and Differential Equations, Marcel Dekker, New York 1976, 1-197.

[7] L. Cesari and R. Kannan, An abstract theorem at resonance, Proc. Amer. Math. Soc. 63 (1977), 221-225.

[8] - - - An existence theorem for periodic solutions of nonlinear parabolic equations, Istit. Lombardo Accad. Sci. Lett. Rend. A 116 (1985), 19-26.

[9] A. Friedman, Partial Differential Equations of Parabolic Type, Prentice-Hall, Englewood Cliffs, N.J., 1964. 
[10] R. Kannan and V. Lakshmikantham, Existence of periodic solutions of semilinear parabolic equations and the method of upper and lower solutions, J. Math. Anal. Appl. 97 (1983), 291-299.

[11] P. J. McKenna, Uniqueness of solutions for semilinear equations at resonance, Nonlinear Anal. 2 (1978), 235-237.

[12] J. Mawhin, Semi-coercive monotone variational problems, Acad. Roy. Belg. Bull. Cl. Sci. (5) 73 (1987), 118-130.

[13] J. J. Nieto, Periodic solutions of nonlinear parabolic equations, J. Differential Equations 60 (1985), 90-102.

[14] - , Nonuniqueness of solutions for semilinear elliptic equations at resonance, Boll. Un. Mat. Ital. (6) 5-A (1986), 205-210.

[15] -, Decreasing sequences of compact absolute retracts and nonlinear problems, ibid. (7) 2-B (1988), 497-507.

[16] T. I. Seidman, Periodic solutions of a nonlinear parabolic equation, J. Differential Equations 19 (1975), 242-257.

DEPARTAMENTO DE ANÁLISIS MATEMÁTICO

FACULTAD DE MATEMÁTICAS

UNIVERSIDAD DE SANTIAGO DE COMPOSTELA

SANTIAGO DE COMPOSTELA, SPAIN 